\title{
Tonal schemas: Evidence obtained by probing distorted musical scales
}

\author{
DANIEL S. JORDAN \\ Xerox Palo Alto Research Center, Palo Alto, Califormia \\ and \\ ROGER N. SHEPARD \\ Stanford University, Stanford, California
}

\begin{abstract}
Listeners were presented with a normal major diatonic scale or with a distortion of that scale obtained either by stretching or by equalizing its intervals. The scale was followed by a test tone, selected from a series of quarter tones spanning the range of the scale, and listeners rated how well the test tone fit in with the scale. The pattern of ratings indicated that listeners interpreted the successive tones of each scale by means of a template-like tonal schema tuned to the usual spacing of the major diatonic scale. For the scale obtained by stretching the major scale to span a semitone more than an octave, the schema evidently shifted monotonically in pitch to accommodate each successive tone. For the scale obtained by making all seven intervals in the scale equal, the schema tended to impose its tonal structure despite the absence of the unequal spacing expected by the schema, and led to illusory impressions that the (usually smaller) third and seventh intervals, though now equal, were larger than the other intervals.
\end{abstract}

The characterization of musical tones solely in terms of their physical attributes, such as frequency, amplitude, and duration, is not sufficient to explain the experience of those tones in a musical context. A musical context tends to activate, within a listener, a particular cognitive schema or mental framework with respect to which ensuing tones are perceptually interpreted. Different contexts, inducing different tonal schemas, may lead to quite different interpretations of the same physical tone.

The musical scales on which the prevailing music of a particular culture is based provide perhaps the most powerful such schemas. In the case of Western listeners, the preeminent such scale is the familiar major diatonic scale, whose successive tones are named do, re, mi, fa, sol, la, $t i, d o$, where the eighth tone, though given the same name as the first $(d o)$, is an octave above it. (Physically, an octave corresponds to a doubling of the fundamental frequency of the tone. The term octave, however, is not based on a physical property but on the musical schema. Within the diatonic musical scale, it is the eighth tone that corresponds to the doubling of frequency.)

The major diatonic scale is characterized by the pattern of intervals between its successive tones within each

This research was supported by the first author's National Institute of Mental Health Traineeship (MH-15157-06) at Stanford University and by the second author's National Science Foundation Research Grants BNS 80-05517 and BNS 85-11685, also at Stanford University. We thank Eddie Kessler for assistance in generating the tone sequences. Correspondence concerning this paper should be sent to Daniel S. Jordan, Intelligent Systems Laboratory, Xerox Palo Alto Research Center, 3333 Coyote Hill Road, Palo Alto, CA 94304, or to Roger N. Shepard, Department of Psychology, Building 420, Stanford, CA 94305. octave, namely, the pattern $2,2,1,2,2,2,1$, where each successive integer indicates the number of semitone steps between adjacent tones of the scale. (A semitone step is, on a physical scale of $\log$ frequency, $1 / 12$ of an octave and on a piano keyboard corresponds to the interval between two adjacent keys-for example, between the white key $\mathrm{C}$ and the next black key $\mathrm{C} \sharp$.) A fundamental property that the major diatonic scale shares with most musical scales is that the pattern of its successive intervals is asymmetric in the sense that as we rigidly shift the whole pattern up or down in pitch, it comes into congruence with itself only after a shift of an integral number of octaves, which, in the case of the Western diatonic system, is a shift of some multiple of 12 semitone steps.

By virtue of this asymmetry, each tone in the scale is distinguished by a unique set of musical intervals to the other tones in the scale. Hence, even though the scale repeats indefinitely over successive octaves (so that an arbitrarily selected portion of successive intervals might exhibit, for example, the pattern ... 2, 1, 2, 2, 1, 2, 2, 2, $1,2,2,1,2, \ldots)$, we can always determine where a given tone is within the scale from the pattern of intervals that separates it from neighboring tones in the scale.

For example, the tone $d o$, called the tonic tone of the scale and regarded as the most important or stable tone (on which pieces of music traditionally end), is unique in that the 3 succeeding tones above it move up by a whole tone, a whole tone, and a semitone (i.e., in the pattern $2,2,1)$, whereas the tone immediately preceding it in the scale is 1 semitone below. Similarly, the tone sol, called the dominant of the scale and generally considered to be the next most stable tone, has the different unique struc- 
tural property that although the 3 succeeding tones above it again move up in the pattern $2,2,1$, the tone immediately preceding it in the scale is 2 semitones (i.e., a whole tone) below.

Every other tone in the scale has a unique such pattern of relations with its neighbors, including tones that are heard as quite unstable and as having a tendency to resolve to a neighboring more stable tone. For example, the tone $t i$, which is called the leading tone because it is heard as having a strong tendency to resolve to the neighboring tone $(d o)$, is the only tone that is preceded in the scale by 3 successive whole-tone steps $(2,2,2)$. The unique structural relations that characterize each tone of a scale are invariant under changes of key. Regardless of whether the scale begins on, say, C, on F, or on $\mathrm{Eb}$ (as when we are in the major keys of $\mathrm{C}, \mathrm{F}$, or $\mathrm{Eb}$, respectively), the leading tone, $t i$ (which would then be $\mathrm{B}, \mathrm{E}$, or $\mathrm{D}$, respectively), will always have the unique property of being preceded by 3 whole-tone steps in the scale of that key.

We have previously proposed that the principal cognitive schema by means of which Western listeners interpret tonal music is a kind of pitch template (Shepard \& Jordan, 1984). The template, being tuned to the pattern of intervals of the major diatonic scale $(2,2,1,2,2,2$, 1), can be shifted up and down in pitch until it comes into register with the pitches of incoming tones. (Often, a default interpretation of the first-presented tone as the tonic will be correct.) When a position is found such that the incoming tones sufficiently match the pitches to which the shifted template is tuned, the tones are categorically assigned their unique tonal functions (tonic, dominant, leading tone, etc.) in accordance with their positions in the template. The categorical nature of this interpretive process is, we suggest, why listeners are often quite insensitive to small mistunings (e.g., Burns \& Ward, 1978; Siegel \& Siegel, 1977; Zatorre \& Halpern, 1979).

In our graphical depiction of the template, we represented the hierarchy of tonal functions by attaching, to each position in the template, a bar whose length is proportional to the importance or stability of that tone (as specified, qualitatively, by music theory or, quantitatively, by experimental measurements about to be described). Thus, the longest bar was attached to the tonic, do, in the template, the next longest bar was attached to the dominant, sol, and so on (see Figure 1 in Shepard \& Jordan, 1984).

This conceptualization represents a direct extension of that of Krumhansl and Shepard (1979), who introduced a probe-tone method for experimentally investigating the hierarchy of tonal functions induced by a musical context. Following the presented context (which, in the initial experiments, was simply a musical scale), a single tone was sounded and the listeners rated that probe tone (e.g., on a 7-point rating scale) according to how well it completed the musical scale. Krumhansl and Shepard found that musical listeners consistently gave higher ratings to tones considered by music theory to be more stable in the presented context. Thus, the tonic was uniformly rated highest, other relatively stable tones such as the dominant and mediant were rated next highest, less stable tones of the scale (including the leading tone) were rated lower, and tones not included in the scale (the chromatic tones between the 7 scale tones) were rated lowest of all. The hierarchy of tonal functions as thus quantified by the probe-tone method is quite robust, having now been replicated in many different studies reported, particularly by Krumhansl, Shepard, and their collaborators (e.g., Kessler, Hansen, \& Shepard, 1984; Krumhansl, 1985; Krumhansl, 1987; Krumhansl \& Kessler, 1982; Krumhansl \& Shepard, 1979).

In the series of experiments reported here, we further explored the idea that the perception of musical tones is mediated by a tonal schema with the properties of a template that can be rigidly shifted in pitch to achieve a fit with the incoming tones. Ordinarily, we are not aware of the role of the hypothesized schema. When music matches the schema-as the preponderance of popular, classical, and folk music in the Western world will-the schema operates so automatically that it is effectively "transparent"' (cf. Shepard, 1981, for visual analogs). The operation of the schema is likely to become evident only when the incoming tones depart from the pattern to which the template is tuned. In this case, the effect of the schema will be to induce some systematic distortions in the relations among the physically presented tones, as they are perceived or remembered. We have explored two distortions of the familiar major diatonic scale.

One distortion that we investigated is a uniform stretching of the major scale in which the relational pattern $(2$, $2,1,2,2,2,1)$ of the semitone intervals remains unchanged but the absolute sizes of the intervals are linearly expanded (on the log-frequency scale) by the ratio 13/12. Hence, the 8th tone of this stretched diatonic scale reaches not just to an octave above its first tone but to a minor 9th above its first tone (i.e., to a semitone beyond the octave). If our notion of the tonal schema as a template is correct, we might expect a slight, and perhaps unnoticed, upward adjustment of the template with each successive, slightly sharper tone, in order to maintain registration of the template with the successive tones of the scale. If so, the adjustment upon the presentation of the final tone would have entailed a shift in key by a semitone (e.g., if the first presented tone was $\mathrm{C}$, from the key of $\mathrm{C}$ major to the key of $\mathrm{C}$ major). Such a shift of the template should reveal itself as a shift, by a semitone, of the entire profile as it is obtained by the probe-tone method.

The second distortion we have explored is an equalization of the diatonic scale, rendering all 7 intervals of exactly the same size on the physical scale of $\log$ frequency. (Such a distortion eliminates, of course, the asymmetry that, as we have noted, is characteristic of virtually all widely used musical scales.) The template theory leads us to expect that the 3rd and 7th intervals of the scale, although of exactly the same physical size as the other intervals in this equalized case, being expected to be physically smaller according to the diatonic pattern $2,2,1$, 
$2,2,2,1$, would be perceived as larger than the other intervals. Moreover, to the extent that the template operates quite automatically in Western listeners, this distortion might emerge even when the listeners are trying to judge the purely physical sizes of the intervals.

Our short initial report of this work (Shepard \& Jordan, 1984) presented preliminary evidence in support of the predictions just outlined. We now present more extensive evidence, based on the profiles of ratings of various probe tones following the presentation of these distorted scales.

\section{EXPERIMENT 1}

In this experiment, we investigated the hypothesized effects of a stretched diatonic scale on the ratings of probe tones following the stretched scale. We constructed the stretched scale by dividing a range of 13 consecutive semitones into 12 equal log-frequency intervals and selecting from the resulting 12 stretched semitones the 7 that corresponded to the tones of the major scale. The interval size between 2 successive tones in this scale deviates from its expected size by only 8.33 cents for semitone intervals and 16.67 cents for whole-tone intervals. However, the accumulated deviation by the eighth tone of the stretched scale amounts to 100 cents (or a semitone), and would normally be quite discriminable (i.e., in an isolated comparison task of 2 tones).

The musical key of a scale is defined by its tonic tone, given by both the first and last tones of the scale, which are, normally, an octave apart in frequency. In the stretched scale, the added difference of a semitone to the normal octave difference between the first and last tones of the scale causes a discrepancy in the assignment of musical key to the scale based on these 2 tones. Will listeners perceive the stretched scale as being in the key of the tonic as given by the starting tone of the scale or as given by the final tone of the scale? Or will they not perceive the stretched scale as a scale at all?

If we assume a rigid template, then the requirement that the tonic tone of this template match both the first and last tones of the stretched scale forces a shift of this template from its initial key to the key a chromatic step above it (in the case of the ascending scale). An alternative possibility is that the template will not rigidly shift but will itself stretch to match the stretched scale.

We presented listeners with both the stretched and normal major scale. Because the majority of the frequencies of stretched-scale tones do not match the frequencies of the normal scale, we needed to interpolate ratings of tones other than those actually probed. Jordan (1987) showed that, using the quarter tones within the frequency range of the musical context, we can interpolate with reasonable accuracy the ratings of all other tones within that range. Therefore, we had listeners judge how well quarter tones within the range of a normal or stretched octave fit in with the musical context generated, respectively, by the normal or stretched scale.

\section{Method}

\section{Subjects}

Twenty-seven Stanford University undergraduates participated in the experiment for credit toward an introductory psychology course requirement. On the basis of their responses to a questionnaire concerning their musical backgrounds (handed out at the beginning of the course), we selected listeners with a wide range of musical backgrounds. Each listener participated in a single 30-min experimental session. One listener reported having absolute pitch and 1 listener returned a response sheet inappropriately marked. These 2 listeners' data are not included in the analyses presented here.

\section{Apparatus}

We generated the musical tones on a 64K Apple II Plus microcomputer operating a MountainComputer Music System synthesizer. The synthezier samples at 32,000 points per second and then filters out all frequencies above $14000 \mathrm{~Hz}$. We used sine waves for all tones, with 30 -msec rise and decay times. Amplification of the filtered tones was equalized to compensate for the effect of the filters. We recorded the analog output on a Sanyo Plus D 55 stereo tape recorder, and during the experimental session played the prerecorded tapes on the same tape recorder.

\section{Stimulus Materials}

We presented listeners with two different types of scales, the normal major and the stretched major scale, both in ascending and descending sequences. We constructed the stretched major scale by dividing a range of 13 (consecutive) semitones into 12 equal log-frequency intervals, and taking from the resulting set of 12 stretched semitones the 7 that corresponded to the 7 in the normal major scale. The equation for the stretched semitone frequencies is

$$
f_{s}(i)=f_{0} R_{s}^{i / 12},
$$

where $f_{0}$ is the frequency of the starting tone of the scale, $f_{s}(i)$ is the frequency of the stretched tone that is $i$ (stretched) semitones away from $f_{0}$ (where $i=1,2,3, \ldots$ for ascending scales and $i=$ $-1,-2,-3, \ldots$ for descending scales) and $R_{s}=2^{13 / 12}$ is the ratio of the frequencies of a stretched octave.

In each trial, we presented listeners with either an ascending or a descending sequence of tones consisting of the $\mathbf{8}$ tones (including the octave) of either a normal or a stretched major scale, followed by a probe tone. In all sequences, the tone duration was $0.5 \mathrm{sec}$, with an intertone interval of $0.05 \mathrm{sec}$. The duration of the probe tone also was $0.5 \mathrm{sec}$, and this tone occurred $1.5 \mathrm{sec}$ after the final tone of the sequence. After each probe tone, we gave listeners $7 \mathrm{sec}$ in which to record their responses before the next trial began.

For variety, we used different frequency ranges. Previous studies have shown that the structure of perceived musical relations is invariant under transposition (e.g., Attneave \& Olson, 1971; Dowling, 1978; Dowling \& Fujitani, 1971). The frequency ranges for the four types of scales were: (1) C-C' for the normal ascending, (2) $\mathrm{D}-\mathrm{D}^{\prime}$ for the normal descending, (3) $\mathrm{D}$ - $\mathrm{E}^{\prime}$ for the stretched ascending, and (4) C-C for the stretched descending scales. Because our analyses required preference ratings of tones between the standard semitone intervals, and because one of us had determined that a probe-tone sampling at quarter tones is sufficient for interpolating the required preference ratings (Jordan, 1987), we probed at quarter tones. Probe-tone sets consisted of the normal quarter tones inclusive within the range of the scale, giving 25 probe tones for the normal major scale and 27 probe tones for the stretched major scale. We randomized the 50 trials of normal scales and divided this set into two blocks. Similarly, we randomized the 54 trials of stretched scales and divided this set into two blocks. We presented listeners with the four blocks of trials in a randomized order. 


\section{Procedure}

Similar to Krumhansl and Shepard (1979), we instructed listeners to rate on a 7-point scale how well the probe tone fit in, musically, with the immediately preceding musical scale. On the rating scale, 1 was marked does not fit at all and 7 was marked fits very well. We encouraged listeners to use the full range of the rating scale, emphasizing that ratings were subjective. We informed them that the scales would be both ascending and descending, would vary in their frequency ranges and, also, that the probe tone would not necessarily be one of the tones heard in the musical scale.

\section{Results \\ Individual Differences}

We had chosen listeners to approximate a uniform distribution of musical backgrounds, which ranged from no background to 16 years of musical instruction and performance. To evaluate the response consistency of listeners with such diverse musical backgrounds, we computed intersubject correlations for the response profiles from both normal- and stretched-scale contexts. These correlations ranged from -.036 to .580 , and averaged .352. Results of applying the additive tree-fitting method, ADDTREE (Sattath \& Tversky, 1977), to the matrix of correlations suggested four major groups of listeners and explained $78 \%$ of the variance in the correlational data (Formula 1 stress $=.086$ ). The groups turned out to differ appreciably in musical background according to the questionnaire. For the four groups, the average numbers of years for performing (instrument or voice) were $2.8,2.8$, 9.8 , and 5.0 and the average numbers of years of musical instruction were, respectively, 2.8, 2.9, 9.6, and 5.0.

To simplify the analyses, we combined groups that did not significantly differ in musical background into two larger groups: Group 1, consisting of the two groups of less musically experienced listeners, and Group 2 , consisting of the two groups of more musically experienced listeners. The average number of years for performing (2.8 for Group 1 and 7.0 for Group 2) differed significantly $[t(23)=2.31, p<.05]$, as did the average number of years of musical training (2.9 for Group 1 and 6.9 for Group 2) $[t(23)=2.61, p<.02]$.

\section{Analysis of Profiles}

Figures 1 and 2 show profiles for Groups 1 and 2. To facilitate comparison of the profiles, the ascending and descending profiles for the normal scale are shown as ranging from $\mathrm{C}$ to $\mathrm{C}^{\prime}$ (a true octave), although actual frequency ranges differed for the two scales. Similarly, ascending and descending profiles for the stretched scale are shown as ranging from $C$ to $C{ }^{\prime \prime}$ (a stretched octave).

Ratings following the normal scale. Listeners' profiles for the normal-scale context were consistent with those obtained in earlier studies, with the more musical listeners more strongly exhibiting the hierarchy of tonal functions expected from music theory (see, especially, Krumhansl \& Shepard, 1979; Kessler et al., 1984). On the semitone level, listeners preferred the 8 diatonic tones $\left(C, D, \ldots, C^{\prime}\right)$ over the 5 nondiatonic tones [for the ascending scales, $t(11)=3.60, p<.01$, and $t(11)=$ $4.24, p<.005$, for Groups 1 and 2, respectively; for the descending scales, $t(11)=4.75, p<.001$, and $t(11)$ $=4.52, p<.001$, for the two groups, respectively]. On the quarter tone level of the hierarchy (Jordan, 1987), listeners preferred the 13 (inclusive) chromatic tones within the octave over the 12 nonchromatic quarter tones $[t(23)=3.31, p<.01$ and $t(23)=3.01, p<.01$, for Groups 1 and 2 , respectively, for the ascending scales; and $t(23)=3.52, p<.005$, and $t(23)=2.70, p<.01$, for the two groups, respectively, for the descending scales.

Ratings following the stretched scale. None of the rating profiles for the stretched-scale context showed evidence of discrimination between any of the levels in the hierarchy of tonal functions. For both groups of listeners and in both ascending- and descending-scale contexts, the rating differences between the diatonic and nondiatonic tones on the semitone level and between the chromatic and nonchromatic tones on the quarter tone levels of the hierarchy were insignificant.

\section{Comparisons of Profiles for Stretched and Normal Scales}

How much do we need to shift and/or stretch the profile for the normal scale to bring it into an optimum fit with the profile for the stretched scale? We first define needed mathematical terms. Letting $x$ refer to tonal frequencies, we denote the rating of a tone heard in the normal- and stretched-scale contexts by $r_{n}(x)$ and $r_{s}(x)$, respectively. Letting $x_{q}$ be $x$ restricted to the quarter tones, we refer to the set of 25 quarter tones spanning a normal octave as $\left\{x_{q}\right\}_{n}$ and the set of 27 quarter tones spanning a stretched octave as $\left\{x_{q}\right\}_{s}$. In this notation, then, $\left\{r_{n}\left(x_{q}\right) \mid x_{q} \in\left\{x_{q}\right\}_{n}\right\}$ and $\left\{r_{s}\left(x_{q}\right) \mid x_{q} \in\left\{x_{q}\right\}_{s}\right\}$ are the sets of rating for the normal- and stretched-scale contexts, respectively, and represent the profiles. We abbreviate and refer to the profiles as $\left\{r_{n}\left(x_{q}\right)\right\}$ and $\left\{r_{s}\left(x_{q}\right)\right\}$.

Our goal is to stretch and/or shift $\left\{r_{n}\left(x_{q}\right)\right\}$, the normalscale profile, so that it best matches $\left\{r_{s}\left(x_{q}\right)\right\}$, the stretchedscale profile. To do this, we define a linear mapping, $t$, on $x$, where $t(x)=\alpha x+\beta$. The term $\alpha$ indicates a scaling (either stretching or contraction) for the tone $x$, and the term $\beta$ represents a translation, or shift. Then, $r_{n}\left(t\left(x_{q}\right)\right)$ $=r_{n}\left(\alpha x_{q}+\beta\right)$ is the rating of a scaled and translated quarter tone in the normal-scale context, and the set $\left\{r_{n}\left(t\left(x_{q}\right)\right)\right\}$ of such ratings is a transformed normal-scale profile. We find values of $\alpha$ and $\beta$ such that the profile $\left\{r_{n}\left(\alpha x_{q}+\beta\right)\right\}$ best matches the stretched profile $\left\{r_{s}\left(x_{q}\right)\right\}$ by using a criterion of maximum correlation $(\varrho)$ of the two profiles. That is, we satisfy:

The profile-matching criterion: Maximize the function $f(\alpha, \beta) \equiv \varrho\left(r_{s}\left(x_{q}\right), r_{n}\left(\alpha x_{q}+\beta\right)\right)$ with respect to the variables $\alpha$ and $\beta$.

As illustrated in the top portion of Figure 3, we study the relationship between $N$ and $S$, the profiles from normal- and stretched-scale contexts, via the transformed normal-scale profile $N_{t}$. To satisfy the profile-matching 


\section{Group 1}
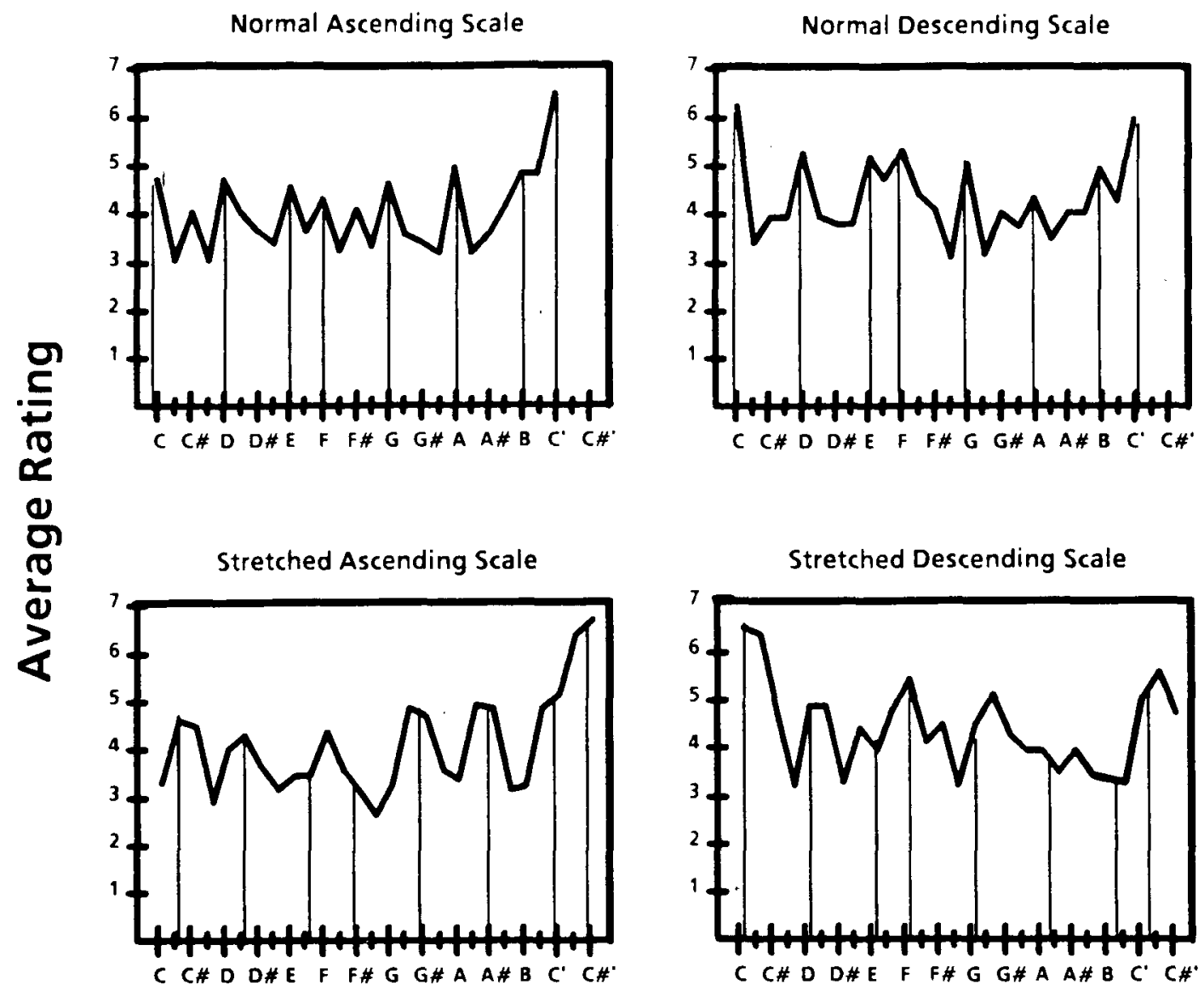

Figure 1. Rating profiles obtained from the more musical listeners (Group 1) in Experiment 1. The results are, for the normal scale (top panels), the stretched scale (bottom panels), the ascending scales (left panels), and the descending scales (right panels). For the normal scale, vertical lines connect the diatonic tones (on the $x$-axis) to their ratings. For the stretched scale, these lines are shifted according to the $\alpha$ and $\beta$ values that maximize the correlation of that profile with its corresponding normal-scale profile.

criterion, we maximize the correlation between ratings in $N_{t}$ and $S$ by varying $N_{t}$. To determine values of $r_{n}$ for tones other than the quarter tones with which listeners were probed, we use the method described by Jordan (1987) and interpolate on the Fourier curve generated by the (original) profile $\left\{r_{n}\left(x_{q}\right)\right\}$; this allows us to estimate ratings for continuous values of $\alpha$ and $\beta$ and, hence, the correlation function $f$.

We note that an alternative approach to this analysis is a profile-matching criterion that maximizes the correlation between ratings from the normal-scale profile, $N$, and an interpolated stretched-scale profile, $S_{t^{-1}}$, as shown in the bottom portion of Figure 3. This approach requires the justification of an interpolation curve (Fourier or otherwise) for the stretched-scale profile. (However, because Jordan, 1987, validated interpolation on Fourier curves for normal-scale profiles only, we follow the approach outlined in the top portion of Figure 3.)

Fourier analyses and determination of parameters $\alpha$ and $\beta$. We performed a Fourier analysis on each of the normal-scale response profiles for the two groups of listeners. To satisfy the requirements for a Fourier analysis, we assumed octave periodicity of the ratings and replaced the two ratings for the endpoints of the octave range with one rating equal to their average. Appendix A gives the amplitude and phase estimates of the 12 harmonics in the Fourier series, along with their percentage contribution to total variance.

We computed the correlation function $f$ such that the translation parameter $\beta$ indicates a shift relative to the starting tone of the (normal) scale; the scaling parameter $\alpha$ indicates a stretch relative to the normal octave inter- 


\section{Group 2}

Normal Ascending Scale

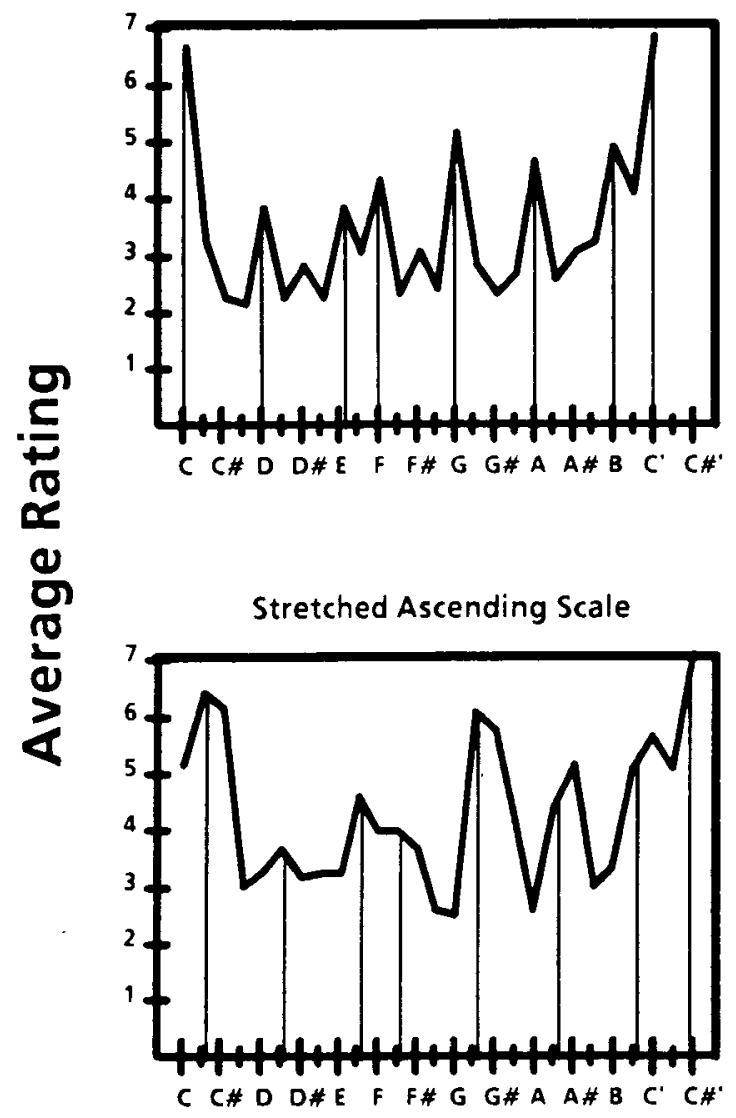

Normal Descending Scale

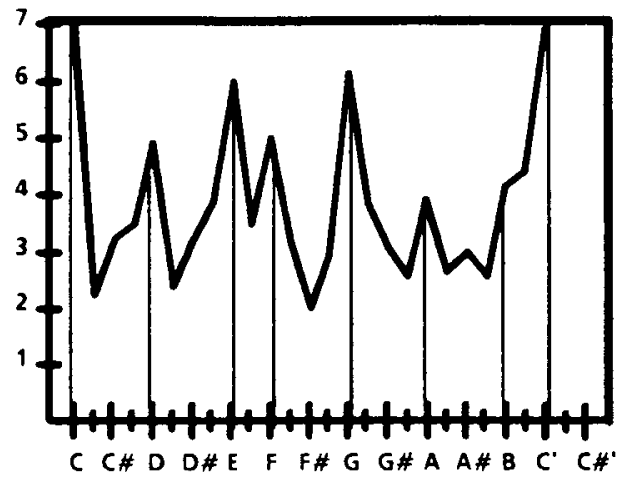

Stretched Descending Scale

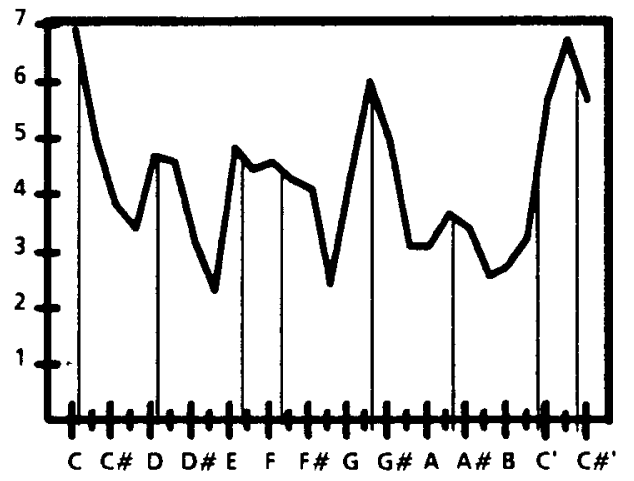

Figure 2. Rating profiles obtained from the less musical listeners (Group 2) in Experiment 1. Again, the results are for the normal scale (top panels), the stretched scale (bottom panels), the ascending scales (left panels), and the descending scales (right panels). Again, for the normal scale, lines connect the diatonic tones (on the $x$-axis) to their ratings, and for the stretched scale, these lines are shifted according to the $\alpha$ and $\beta$ values that maximize the correlation of that profile with its corresponding normal-scale profile.

val. $\beta$ is in units of semitones. Figure $4 \mathrm{a}$ illustrates that a linear mapping with a $\beta$ value of 1.0 produces a semitone shift of tones. Figure $4 \mathrm{~b}$ illustrates that a linear mapping with an $\alpha$ value of $13 / 12=1.083$ produces a semitone stretch of tones over an octave span. In these two examples, the corresponding normal-scale profiles for these quarter tones would be, respectively, shifted and stretched by a semitone. The assumption of (normal) octave periodicity of the ratings permits us to interpolate the ratings of tones that fall outside the octave range spanned by the quarter tone probes. For example, we may map a tone, say $C{ }^{\prime}$, from the stretched-scale context onto a (micro)tone in the normal-scale context that is higher than the highest tone $\left(C^{\prime}\right)$ probed in the normal-scale context by assigning it the rating for $\mathrm{C}$ an octave below it.

We employed numerical methods to find the maximum of the correlation function $f$ with respect to $\alpha$ and $\beta$, and used a hill-climbing technique, STEPIT (Chandler, 1969). Table 1 shows the solutions for $\alpha$ and $\beta$ for both groups of listeners and both ascending- and descending-scale contexts, along with their corresponding maximum correlation. For comparison, Table 2 gives the correlation for special cases of $\alpha$ and $\beta$. In these tables, values of $\alpha$ and $\beta$ are also given in units of cents. (Also, Figures 1 and 2 show vertical lines from the diatonic tones to their ratings that for the stretched scale are shifted according to these values of $\alpha$ and $\beta$.)

For the ascending scale, the correlation function for Group 1 listeners achieves a maximum of 0.674 in the ascending-scale context for $\alpha$ and $\beta$ equal to 1.008 and 0.800 , respectively. These parameter values translate into a tonal stretch of 9.6 cents and a tonal shift of 80 cents for the normal-scale profile. By comparison, with no shifting or stretching of the normal profile, the correlation be- 

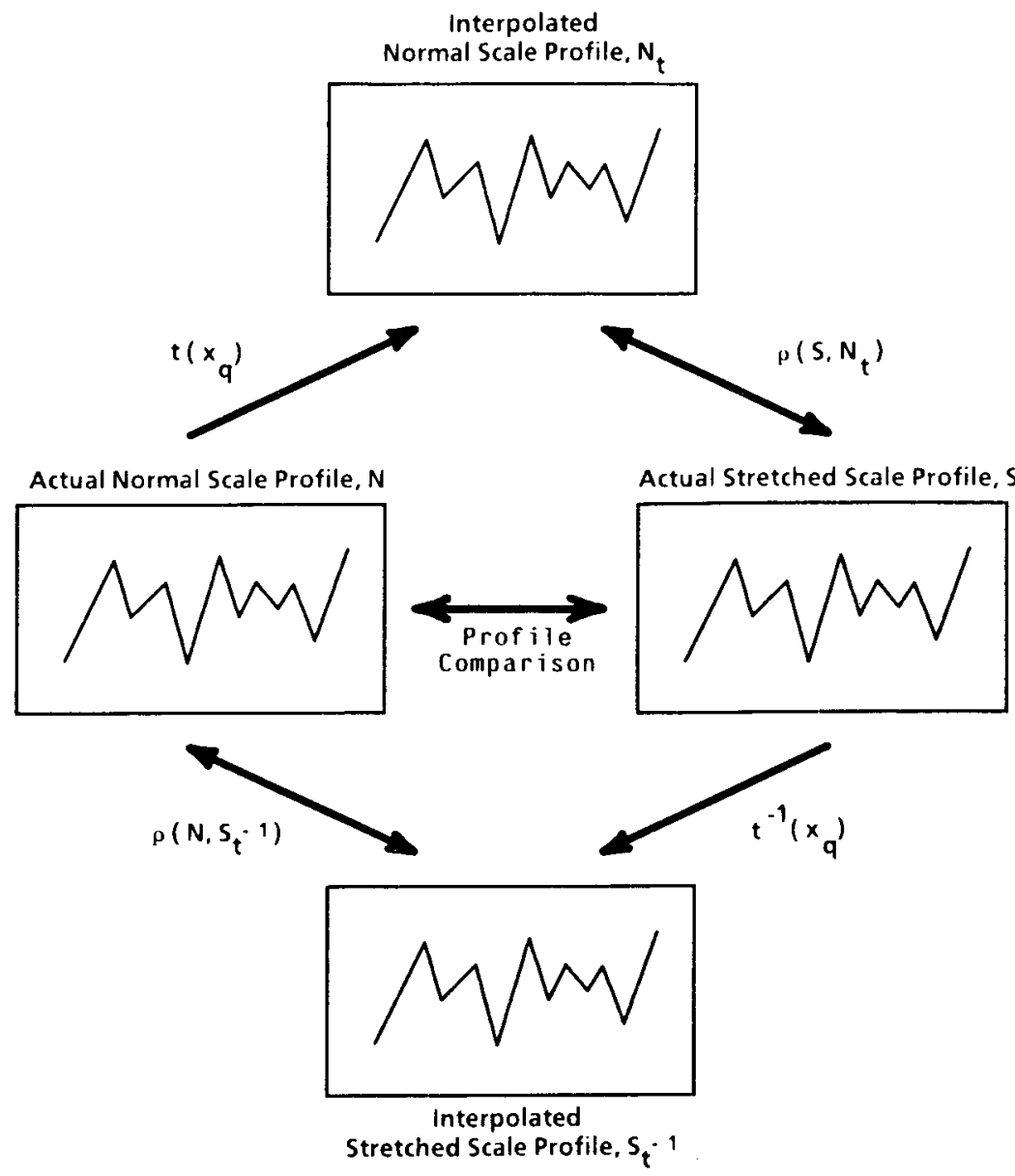

Figure 3. A diagram of the analyses for comparing profiles for the normal and stretched scales. In the top portion of the figure, the ratings from $S$, the stretched-scale profile, are correlated with ratings from $N_{t}$, the profile of the normal scale $N$ transformed by the linear transformation $t$ (whose domain is the quarter tones, $x_{q}$ ). In the bottom portion of the figure, an alternative inverse approach correlates the ratings from $N$ with ratings from $S_{t^{-1}}$, the profile of the stretched-scale $S$ transformed by the inverse linear transformation $t^{-1}$.

tween the normal and stretched profiles is -0.085 . For Group 2 listeners, the correlation function achieves a maximum of 0.893 in the ascending scale context for $\alpha$ and $\beta$ equal to 1.010 and .740 , respectively. These parameter values translate into a tonal stretch of 12 cents and a tonal shift of 74 cents. By comparison, with no shifting or stretching of the normal profile, the correlation between profiles is $-\mathbf{0 . 0 4 7}$. Thus, for both groups of listeners, in the ascending-scale context the translational component $\beta$ strongly dominates the transformation of the normalscale profile, varying from $3 / 4$ to $4 / 5$ of a semitone, and any scaling effect as given by $\alpha$ is minimal.

For the descending scale, the correlation function for Group 1 listeners achieves a maximum of 0.608 for $\alpha$ and $\beta$ equal to 1.004 and 0.670 , respectively. These parameter values translate into a tonal stretch of 4.8 cents and a tonal shift of 67 cents for the normal-scale profile. By comparison, with no shifting or stretching of the normal profile, the correlation between profiles is 0.254 . For
Group 2 listeners, the correlation function $f$ achieves a maximum of 0.817 for $\alpha$ and $\beta$ equal to 1.053 and 0.165 , respectively. These parameter values translate into a tonal stretch of 63.6 cents and a tonal shift of 16.5 cents. By comparison, with no shifting or stretching of the normal profile, the correlation between profiles is 0.363 . Thus, the relative influence of $\alpha$ and $\beta$ on the transformation of the normal profile differs for the two groups of listeners. For Group 1, $\beta$ dominates the transformation of the normal-scale profile and indicates a profile shift of $2 / 3$ of a semitone. For Group 2, however, the scaling component $\alpha$ dominates, and indicates a profile stretch of almost $2 / 3$ of a semitone.

\section{Discussion}

The principal new finding of Experiment 1 can be stated as follows: Following the presentation of a major diatonic scale that had been linearly stretched to extend 1 semi- 


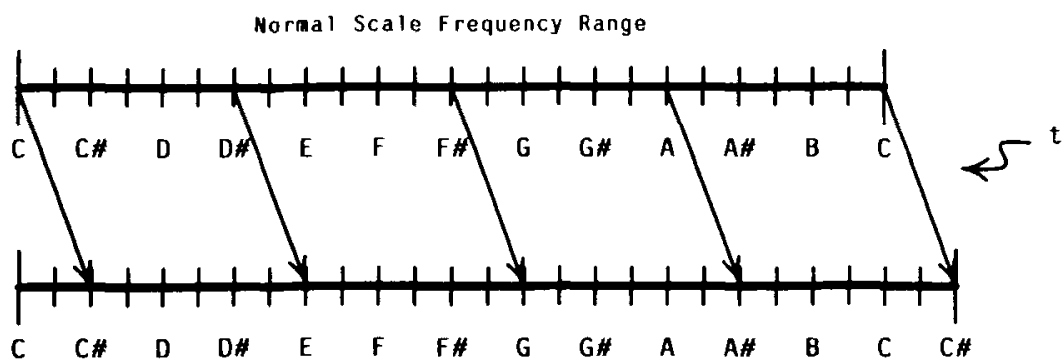

Stretched Scale Frequency Range

b Semitone Stretch

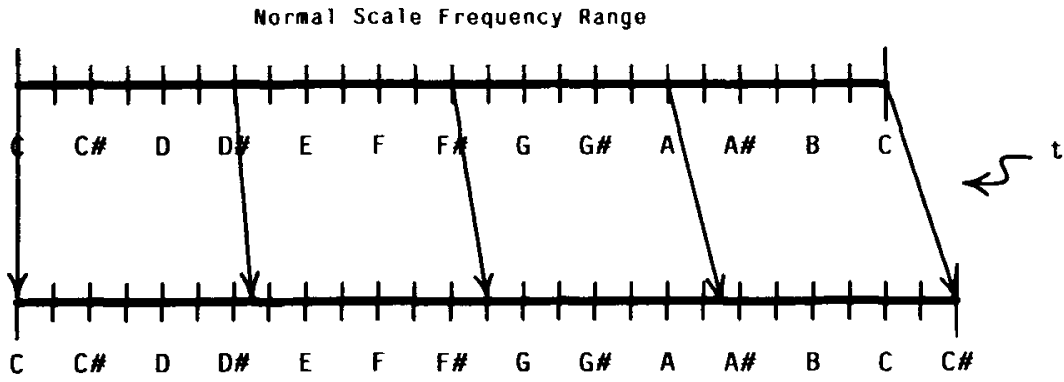

Stretched Scale Frequency Range

Figure 4. Dlustration of the mapping of tones from the normal-scale frequency range to the stretched-scale frequency range by the linear transformation $t$ for (a) a shift of 1 semitone (with no stretching), and (b) a stretch of 1 semitone over the octave range (with no shifting). Corresponding values of $\alpha$ and $\beta$ are (a) 1.0 (0 cents) and 1.0 (100 cents), respectively, and (b) $13 / 12=1.083$ (100 cents) and 0 ( 0 cents), respectively.

tone beyond the usual octave, rating profiles obtained by the probe-tone method of Krumhansl and Shepard (1979), although exhibiting some evidence of a slight stretching, were primarily translated in pitch-by the major part of a semitone. Thus the tonal schema by means of which listeners interpreted the tones, although perhaps slightly adjustable in length, appears to have acted primarily like a template that shifted up or down in pitch, as a semirigid whole, to maintain registry with each succeeding tone of the scale.

The question might be raised, however, as to whether the template that we have posited really does play an essential role in the perception and memory of tones, or whether it is merely the manifestation of the particular rating method used. It could be that the ratings were all made on the basis of interval relations to the last tone of the scale and, yet, that listeners still retained an accurate, undistorted memory for the tones actually sounded at the beginning of the scale. In an additional experiment, which we reported earlier (Shepard \& Jordan, 1984), we obtained evidence supportive of the claim that following the same stretched scale, even the memory for the first tone of the scale was distorted by the shift that we have posited in the tonal template.
In that experiment, we presented the ascending and descending stretched scales as in Experiment 1 here, except that we gave the 57 listeners $10 \mathrm{sec}$ in which to respond before the next trial began. Following the scale, listeners heard either a target test tone that was the original starting tone of the stretched scale (and 13 semitones away from the last tone of the scale) of a decoy test tone that was 1 semitone removed from the starting scale tone (and 12 semitones away from the last tone in the scale). The listeners' task was to decide whether or not the test tone was the starting tone of the scale. Listeners responded

Table 1

Maximum Correlations of Profiles in Experiment 1 (With Values of $\alpha$ and $\beta$ )

\begin{tabular}{lccc}
\multicolumn{1}{c}{ Scale } & $\varrho_{\max }$ & $\alpha$ (cents) & $\beta$ (cents) \\
\hline & & Group 1 & \\
Ascending & .674 & $1.008(9.6)$ & $0.800(80.0)$ \\
Descending & .608 & $1.004(4.8)$ & $0.670(67.0)$
\end{tabular}

Group 2

\begin{tabular}{llll} 
Ascending & .893 & $1.010(12.0)$ & $0.740(74.0)$ \\
Descending & .817 & $1.053(63.6)$ & $0.165(16.5)$ \\
\hline
\end{tabular}

Note-Correlation function is $\varrho\left(r_{s}\left(x_{q}\right), r_{n}\left(\alpha x_{q}+\beta\right)\right)$. 
Table 2

Correlations ( $e$ ) of Profiles in Experiment 1 for Boundary Values of $\alpha$ and $\beta$

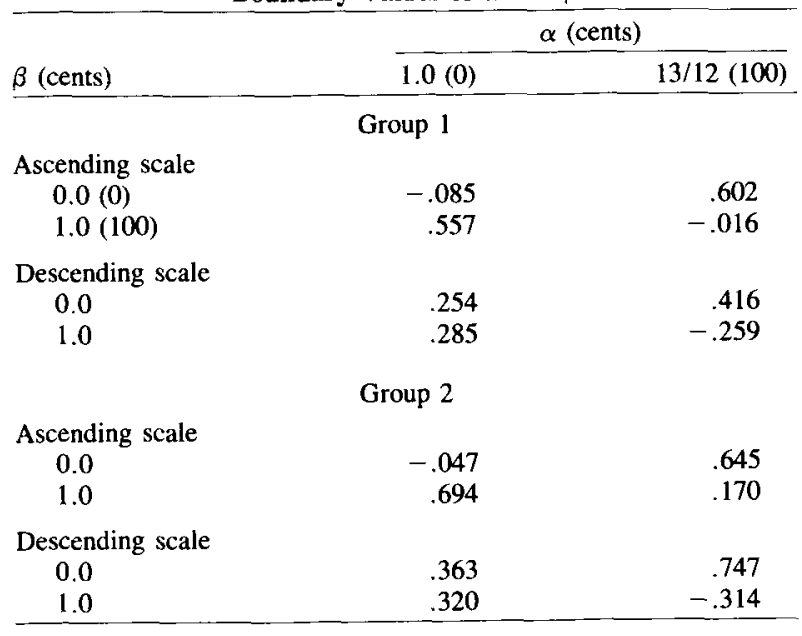

by marking a 5-point rating scale that was numbered from -2 to +2 and labeled definitely too low, probably too low, probably the same, probably too high, and definitely too high. (To minimize transfer, we used different frequency ranges for the four scales; however, for convenience in reviewing the results here, we will refer to the ranges for all four scales as if they were $\mathrm{C}-\mathrm{C} \sharp^{\prime}$.)

For the ascending scale, the target test tone (C), which was the first tone of the sequence, was rated as lower than that scale's starting tone by $86 \%$ of the listeners, with $48 \%$ rating it as definitely lower. Only $9 \%$ of the listeners correctly rated that tone as the same as the starting tone. The tendency to rate the target test tone as lower than the starting tone (when, in fact, it was the starting tone) was highly significant $(p<.0001)$. In contrast, the decoy test tone $\left(\mathrm{CH}^{\sharp}\right)$, a semitone above the first tone of the scale $(\mathrm{C})$ and an octave below the last tone of the scale, was rated by $63 \%$ of the listeners as being the same as the starting tone. Only $11 \%$ of the listeners correctly rated this test tone as higher than the starting tone.

Listeners' memories of the starting tone were similarly, though somewhat less markedly, distorted for the descending scale. Sixty-eight percent of the listeners rated the target test tone, $C{ }^{\prime}$ ' (the first tone of the scale), as higher than that scale's starting tone, with $28 \%$ rating it as definitely higher. Only $25 \%$ of the listeners correctly rated that tone as the same as the starting tone. The tendency to rate the target tone as higher than the starting tone was highly significant $(p<.0001)$. In contrast, the decoy test tone $\left(C^{\prime}\right)$ was rated by $49 \%$ of the listeners as being the same as the starting tone (when, in fact, it was a semitone below it). Only $15 \%$ of listeners correctly rated the decoy test tone as lower than the starting tone of the scale.

Evidently, then, listeners' memories for the starting tone of a stretched scale were affected by the shift in the tonal schema that was induced by the stretched scale. Moreover, this memory shift of the first tone toward the decoy tone was more pronounced for the ascending than for the descending scale. This asymmetry is consistent with the findings of Experiment 1, in which the profile shift of the first tone for the ascending scale ranged from 74 to 80 cents-bringing it very close to the decoy tone--while this tone's shift for the descending scale ranged from 17 to 67 cents, a smaller and less consistent shift.

Because Experiment 1 showed that listeners' perceptions of tones in the entire octave range were distorted, we discount the alternative hypothesis for this further $\mathrm{ex}$ periment that listeners were merely judging the octave relationship of the test tone to the last tone of the scale. Together, results from these two experiments suggest that a template-like diatonic tonal schema strongly influences the perception and memory of pitches.

\section{EXPERIMENT 2}

In this experiment, we investigated the effects of a different kind of distortion of the major diatonic scale. Instead of linearly stretching all seven intervals so that the resulting scale spanned more than an octave, we adjusted the individual intervals so that they were all exactly equal, on the physical continuum of log frequency, while the whole scale still spanned exactly the octave of the normal scale.

As we have noted, the major diatonic scale is characterized by a particular, asymmetric pattern of intervals-in terms of numbers of semitones making up each successive interval: 2, 2, 1, 2, 2, 2, 1. As we also indicated, some such asymmetric pattern is characteristic of the traditional musical scales of most cultures (see, e.g., Catellano, Bharucha, \& Krumhansl, 1984; Dowling, 1978; Kessler et al. 1984), and has the musically and cognitively significant property that a listener can always tell where any given tone in a sequence falls within the implied tonal structure (Balzano, 1980; Shepard, 1982). Although 20th century composers have experimented with scales that have equal intervals (such as the whole-tone scale of Debussy or the 12-tone scale of Schoenberg), popular, classical, and folk musics seem never to have made wide use of such scales. Because of their complete symmetry, such scales provide no fixed framework with respect to which the music can move or come to rest, with respect to which tones can be assigned a hierarchy of unique tonal functions, or with respect to which there can be a sustained buildup of tension followed by final resolution.

What happens, then, when a scale is played that completely lacks the usually expected asymmetric pattern of sizes of successive intervals but has the same number of tones as a major diatonic scale? We might suppose that listeners will still manifest some tendency to impose their internalized, more structured, and asymmetric tonal schema upon such an unstructured and symmetric input. If so, the probe-tone method should provide some evidence, at least for the more musically experienced listeners, of the imposition of the standard tonal hierarchy (tonic, dominant, mediant, etc.). The imposition of such a tonal schema should also lead to perceptual illu- 
sions and errors of memory in which the third and seventh intervals, which are normally smaller (according to the pattern $2,2,1,2,2,2,1$ ) but which are rendered equal in the distorted scale, might seem, by reference to the schema, to be larger than the other intervals.

\section{Method}

\section{Subjects}

Nineteen Stanford University undergraduates participated in the experiment, receiving credit toward an introductory psychology course requirement. As in Experiment 1, we preselected listeners with a range of musical backgrounds, on the basis of their responses to a questionnaire handed out at the beginning of the course. Each listener participated in a single 45 -min experimental session. The data for one listener, who reported having absolute pitch, are not considered here.

\section{Apparatus}

The stimuli were synthesized, recorded, and played to the listeners on the equipment already described in Experiment 1.

\section{Stimulus Materials}

We presented listeners with two different types of scales, the normal major scale and the 7-interval equalized scale, both in ascending and descending sequences. We constructed the tones for the equalized scale by dividing an octave into 7 equal log-frequency intervals; we refer to the resulting 7 tones as equalized scale tones. The equation for these tones is

$$
f_{E}(i)=f_{0} R^{\prime \prime \prime},
$$

where $f_{E}(i)$ is the frequency of the tone in the equalized scale that is $i$ equalized scale tones away from the starting tone $f_{0}$ (where $i$ $=1,2,3, \ldots$ for ascending scales and $i=-1,-2,-3, \ldots$ for descending scales), and $R=2$ is the ratio of the frequencies of an octave.

As in Experiment 1, each trial consisted of a sequence of 8 tones followed by a probe tone that listeners rated. The tone and intertone durations were the same as in that experiment, with the listeners being given $7 \mathrm{sec}$ in which to respond before the next trial began. The listeners rated one set of probe tones for the normal major scale context and two sets of probe tones for the equalized scale context. For the normal-scale context, probe tones consisted of the 25 (inclusive) quarter tones within an octave. For the equalized-scale context, one set of probe tones consisted of this same set of 25 quarter tones. A second set of probe tones consisted of tones separated by intervals one-half the size of the intervals between the equalized scale tones; we refer to those tones as equalized semiscale probes. These tones are obtained by dividing the octave into 14 equal logfrequency intervals. The equation for these tones is

$$
f_{E}(i)=f_{0} R^{i / 14} \text {. }
$$

As in Experiment 1, we varied frequency ranges for variety. Frequency ranges for the six different scale/probe tone cases were: (1) $C^{\prime}-C$ for the normal descending scale; (2) $D E-D \#^{\prime}$ for the normal ascending scale; (3) $D^{\prime}-D$ for the equalized descending scale with quarter-tone probes; (4) $\mathrm{CH}-\mathrm{C}$ ' for the equalized ascending scale with quarter tone probes; (5) $D{ }^{\prime \prime}-D$ for the equalized descending scale with equalized semiscale tone probes; and (6) C-C' for the equalized ascending scale with equalized semiscale probes. We randomly ordered the $\mathbf{5 0}$ trials for the normal-scale context and divided them into two equal-sized blocks. Similarly, we randomly ordered the 50 trials for the equalized-scale context with quarter tone probe tones and the 30 trials for the equalized-scale context with equalized semiscale probes within their respective contexts, and divided each set into two equal-sized blocks for a total of four blocks. We then randomly ordered the six blocks of trials from these three context/probe-tone combinations and presented them to the listeners.

\section{Procedure}

The procedure was identical to that used in Experiment 1.

Results
Individual Differences
As in Experiment 1, we chose listeners to approximate a uniform distribution of musical background, which in this experiment ranged from no background to 18 years of musical instruction and performance. We again evaluated the response consistency of listeners by computing intersubject correlations for response profiles from the normal- and both equalized-scale contexts and submitting these correlations as similarity measures to the ADDTREE tree-fitting algorithm (Sattath \& Tversky, 1977). Correlations ranged from -.019 to .689 , and averaged .281. The ADDTREE solution, which explained $87 \%$ of the variance in the correlational data (Formula 1 stress $=.078$ ), suggested three groups of listeners. Again, the groups turned out to differ appreciably in musical background. For the three groups, the average numbers of years for performing (instrument or voice) were 6.8, 10.0, and 2.1 , and the average numbers of years of musical instruction were, respectively, $7.2,11.5$, and 2.5 .

To simplify the analyses, we combined the two groups of more musically experienced listeners (who did not differ significantly in musical background) into a single Group 1 . The less musically experienced listeners then became Group 2. The 9 listeners in Group 1 differed significantly from the 9 listeners in Group 2 on both measures of musical background [for years of performing an instrument, $t(16)=3.97, p<.005$, for 8.2 vs. 2.1 years, respectively; and for years of musical training, $t(16)=$ $3.51, p<.01$, for 9.1 vs. 2.4 years, respectively].

\section{Analysis of Profiles}

Figures 5 and 6 show profiles for Groups 1 and 2 for each of the three different scale/probe-tone contexts. In these figures, all profiles are displayed, for convenience, as if ranging from $C^{\prime}$ to $C^{\prime}$, although actual frequency ranges for the scales differed.

Ratings following the normal scale. Listeners' profiles for the normal scale were again consistent with those obtained in earlier studies, with the more musical listeners more strongly exhibiting the hierarchy of tonal functions. On the semitone level, listeners preferred the 8 diatonic tones $\left(\mathrm{C}, \mathrm{D}, \ldots, \mathrm{C}^{\prime}\right)$ over the 5 nondiatonic tones [for the ascending context, $t(11)=7.05, p<.001$, and $t(11)$ $=2.50, p<.025$, for Groups 1 and 2, respectively; for the descending scale context, $t(11)=9.04, p<.001$, and $t(11)=2.49, p<.025$, for the two groups, respectively]. On the quarter tone level of the hierarchy, listeners preferred the 13 (inclusive) chromatic tones within the octave over the 12 nonchromatic quarter tones [for the ascending scale context, $t(23)=3.66, p<.001$, and 


\section{Ascending Scales}

\section{Group 1}

Normal Scale

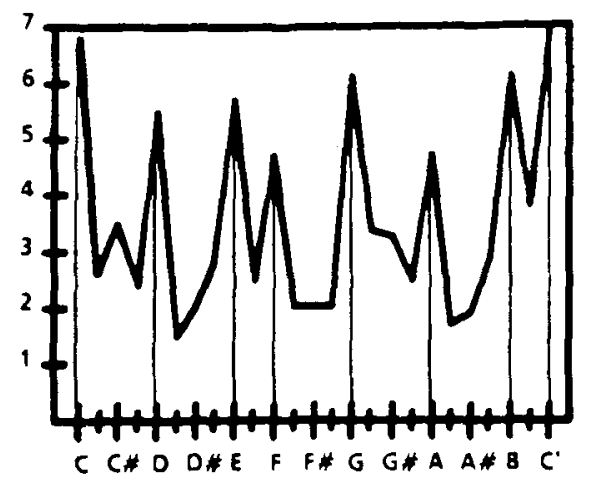

Equalized Scale

(quartertone probes)

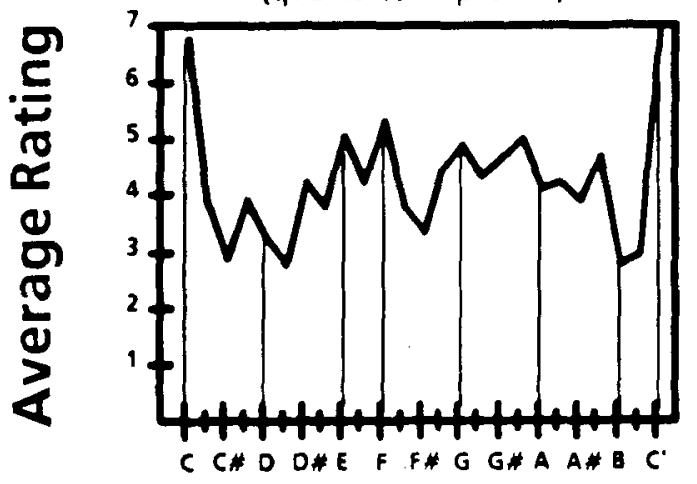

Equalized Scale

(semi-scale probes)

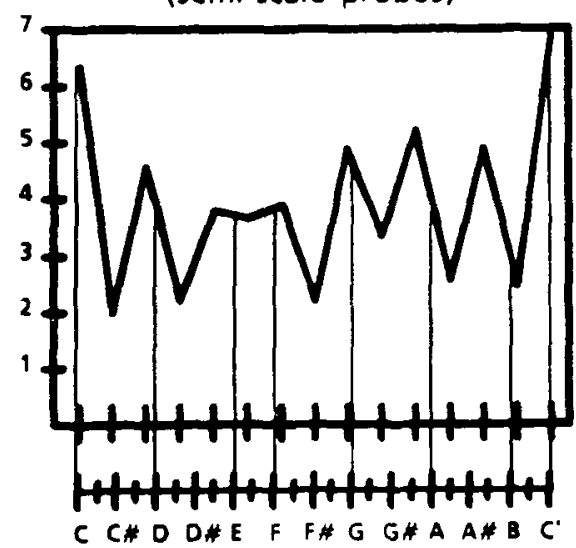

Group 2

Normal Scale

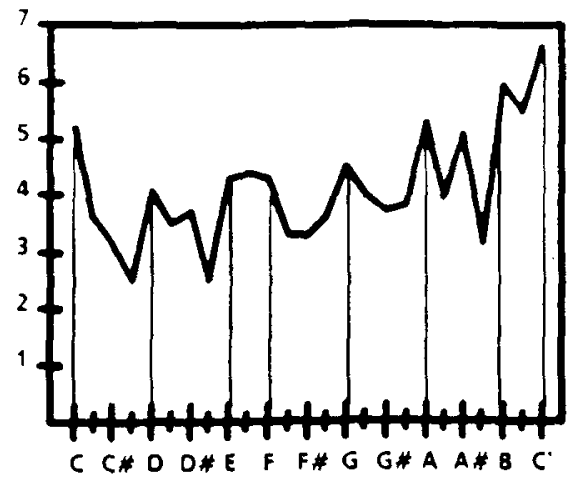

Equalized Scale (quartertone probes)

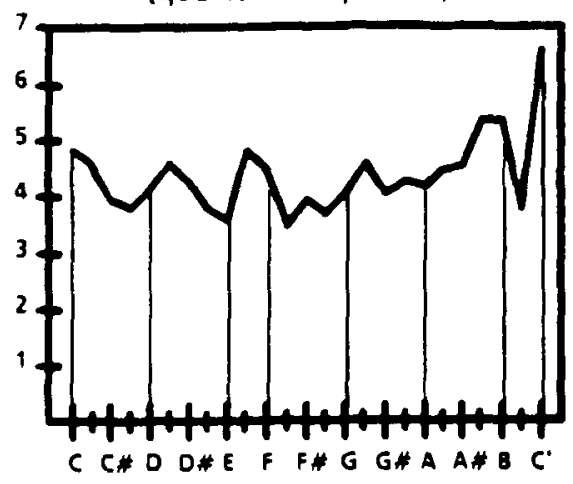

Equalized Scale (semi-scale probes)

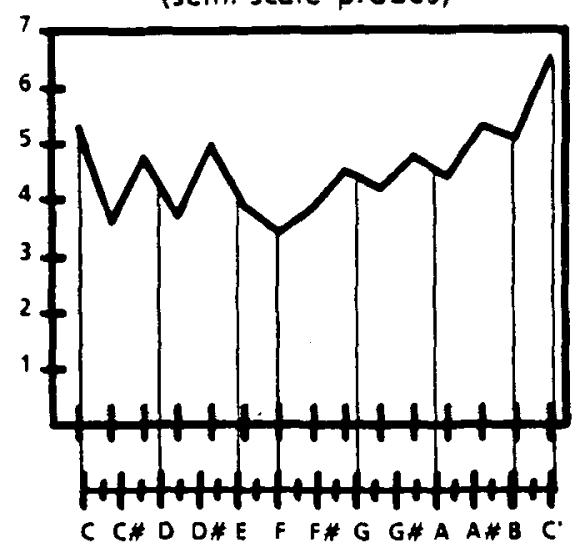

Figure 5. Rating profiles obtained for the ascending scale in Experiment 2. The results on the left are from the more musical listeners (Group 1); those on the right are from the less musical listeners (Group 2). In each case, the top panel is for the normal scale and the two lower panels are for the equalized scale-obtained with quarter tone probes (middle) or with semiscale probes (bottom). In the top two panels, vertical lines connect the diatonic tones to their ratings; in the bottom panel they are shown for comparison with the semiscale probes. 


\section{Descending Scales}

\section{Group 1}

Normal Scale

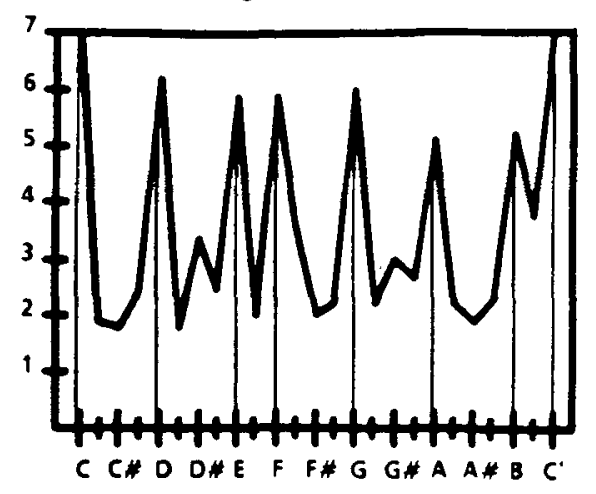

Equalized Scale

(quartertone probes)
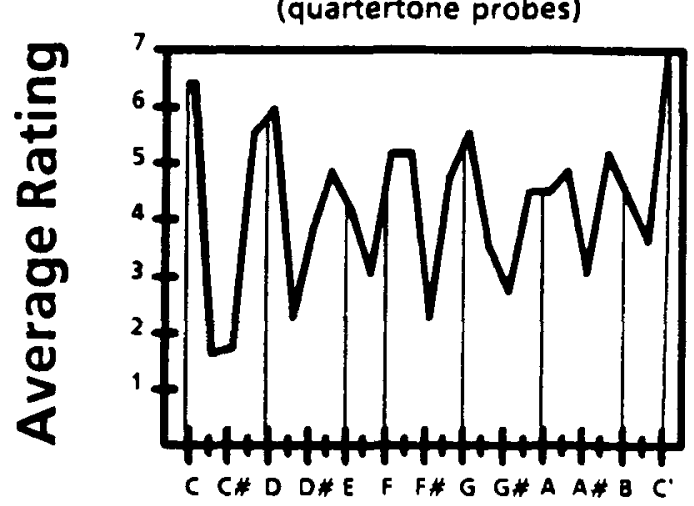

Equalized Scale

(semi-scale probes)

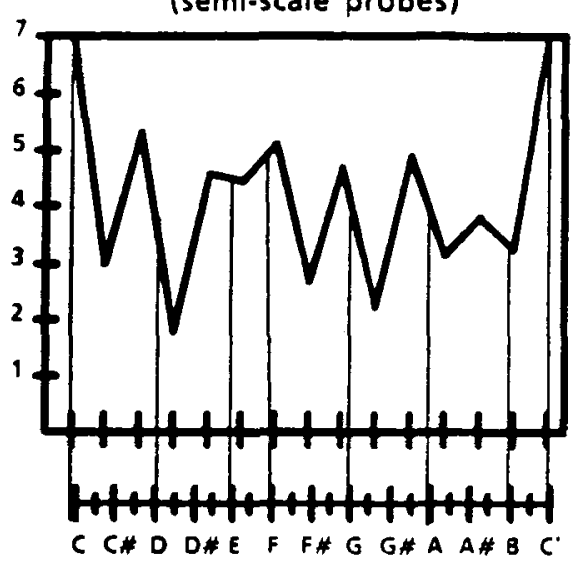

Group 2

Normal Scale

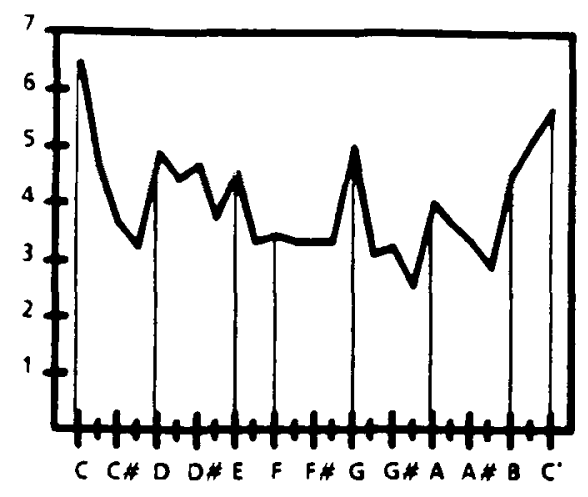

Equalized Scale (quartertone probes)

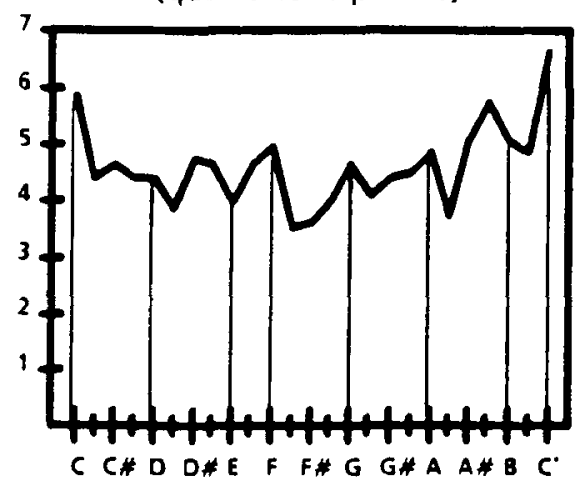

Equalized Scale (semi-scale probes)

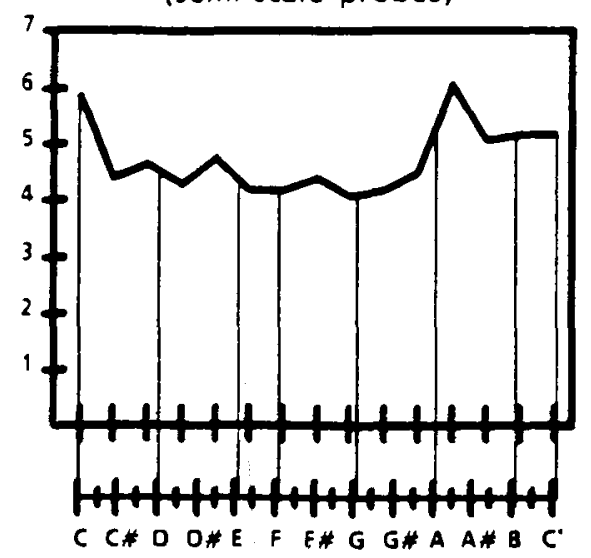

Figure 6. Rating profiles obtained for the descending scale in Experiment 2. Again, the results on the left are from the more musical listeners (Group 1) and those on the right are from the less musical listeners (Group 2). In each case, the top panel is for the normal scale and the two lower panels are for the equalized scaleobtained with quarter tone probes (middle) or with semiscale probes (bottom). In the top two panels, vertical lines connect the diatonic tones to their ratings; in the bottom panel they are shown for comparison with the semiscale probes. 
$t(23)=2.40, p<.025$, for Groups 1 and 2 , respectively; for the descending scale context, $t(23)=3.67, p<.001$, and $t(23)=2.10, p<.025$, for the two groups, respectively.]

Ratings following the equalized scale. The quartertone probes of the equalized scale (which do not coincide with the tones in the equalized scale) provided evidence for the imposition of the usual tonal hierarchy in the case of the more musical listeners (Group 1), but this was statistically significant only for the context of the descending scale, in which case these listeners strongly preferred the 8 diatonic tones over the 5 nondiatonic tones [t(11) $=4.97, p<.001]$.

Listeners were discriminating in their preferences of the 15 equalized-semiscale probes in the equalized-scale context in a manner analogous to the distinction made between diatonic and nondiatonic probes in the normal-scale context. Group 1 listeners preferred the 8 equalizedsemiscale probes that occurred in the equalized scale over the 7 that did not $[t(13)=5.17, p<.001$, and $t(13)=$ $4.56, p<.001$, for the ascending and descending scales, respectively]. Group 2 listeners exhibited this preference of probes for the ascending scale only $[t(13)=2.22$, $p<.05]$.

\section{Comparison of Equalized and Normal Scale Profiles}

We performed analyses to compare the normal-scale profile with the two equalized-scale profiles obtained with different probe-tone sets. For the equalized-scale profile obtained with quarter-tone probes, we directly correlated preference ratings from the equalized-scale context with preference ratings from the normal-scale context. These correlations are given in Table 3. For Group 1 listeners, descending-scale profiles correlated better than the ascending-scale profiles. For Group 2 listeners, ascending-scale profiles correlated better than the decending-scale profiles (although the correlations were generally lower for Group 2).

To compare the profile for the equalized scale obtained with equalized-semiscale probes with the profile for the normal-scale profile obtained with quarter-tone probes, we estimated the ratings for the equalized-semiscale probes in the normal-scale context by interpolating on the Fourier curve generated by the normal-scale profile. Appendix $B$ gives the amplitude and phase estimates for the 12 Fourier harmonics along with their percentage contribution to total variance. The correlations between the interpolated equalized-semiscale probes for the normal-scale context and the (actual) equalized-semiscale probes for the equalized-scale context are given in Table 3, for both ascending and descending sequences. For Group 1 listeners, the correlations were, for both ascending and descending cases, greater than corresponding correlations between equalized-and normal-scale profiles with quartertone probes. For Group 2 listeners, however, these correlations showed a decrease from corresponding correlations between profiles with quarter-tone probes.
Table 3

Correlations of Profiles in Experiment 2

\begin{tabular}{lcc}
\hline & \multicolumn{2}{c}{ Scale } \\
\cline { 2 - 3 } Listeners & Ascending & Descending \\
\hline & Using Quarter-Tone Probes \\
Group 1 & .477 & .689 \\
Group 2 & .574 & .444 \\
& & \\
Group 1 & Using Equalized Semiscale Probes* \\
Group 2 & .633 & .805 \\
\hline
\end{tabular}

*Ratings for these probes in the normal scale context were interpolated.

\section{Discussion}

Experiment 2 has provided evidence that musically sophisticated listeners, anyway, tend to impose the diatonic tonal template, with its associated hierarchy of tonal functions, on a set of tones-even when those tones are equally spaced within an octave and, so, do not conform to the asymmetric pattern characteristic of the normal diatonic scale. These results are reminiscent of the crosscultural results reported by Kessler et al. (1984), in which Balinese listeners tended to impose a tonal hierarchy on their Slendro scale-a 5-tone scale with intervals that do not differ very markedly from equality.

As in Experiment 1, the question might again be raised, however, as to whether the ratings of fittingness of the probe tones reflect any distortions that the imposition of the tonal schema may have caused in the actual perception or memory of the presented tones. In our previously reported experiments (Shepard \& Jordan, 1984), we investigated this matter with the same equalized scale used in Experiment 2 here. Specifically, rather than having listeners make comparisons between the tones of the equalized scale and a subsequent probe tone, we had listeners make comparisons between the intertone intervals within the equalized scale itself.

Two groups of subjects participated in two different versions of that experiment, using the ascending sequence of tones from Experiment 2. We played the scale three times, the first time at a fairly rapid rate to enable the listeners to become familiar with the scale, and the last two times at a slower rate (in which the tone and intertone durations were 0.5 and $1.20 \mathrm{sec}$, respectively) so that the listeners could mark their responses. The frequency range of the scale was always $\mathrm{C}-\mathrm{C}^{\prime}$.

In the first version of the experiment, we instructed 43 listeners to judge the physical size of each interval they heard in comparison to the other intervals. In the second version, we instructed 41 listeners to judge each interval in comparison to the immediately preceding interval. For both versions, listeners expressed their judgments of the intervals as being "larger," "smaller," or "the same" by marking a,+- , or 0 , respectively, on lines that separated the tones, which were shown as small circles, in a pictorial representation of the successive tones. In the first version of the experiment, listeners rated all 7 inter- 
vals; in the second version, the first interval (having no preceding interval) could not be rated. In both versions, we instructed the listeners, who had just learned about the physical measure of $\log$ frequency of tones in a course on perception, to base their judgments on the physical sizes of the intervals only.

For both groups of listeners, the 3rd and 7th intervals in the equalized scale, which correspond to the two smaller (i.e., semitone) intervals in the normal major scale, were the intervals most frequently rated as larger. Thirty-three percent of listeners in the first version and 59\% of listeners in the second version judged the third interval as larger, whereas only $12 \%$ and $2 \%$ of these listeners, respectively, judged the interval as smaller. An even greater percentage of listeners judged the 7 th interval as larger, $72 \%$ and $63 \%$, in the two versions, respectively, with only $7 \%$ in both groups judging the interval as smaller. Contrasts designed to test these differences were highly significant ( $p<.001$ in all cases).

Evidently, then, the musical schema of the diatonic scale tends to influence the actual perception and memory of tones (1) even when those tones are equally spaced in log frequency and, so, do not correspond to a musical scale, and (2) even when the listeners are asked to judge the tones with respect to their physical relationships (on the log-frequency continuum) and not with respect to any musical relationships. In particular, the $3 \mathrm{rd}$ and 7 th intervals in the equally spaced scale are judged larger than the other intervals, even though equal, because the major diatonic template finds those intervals to be too large with respect to the smaller (semitone) intervals to which it is tuned in those positions. The perceptual distortion is greatest, moreover, in the 7th position-probably because the expectation of the semitone interval is especially strong in the step from the leading tone back to the tonic.

\section{CONCLUSIONS}

We take the results reported here and in our earlier paper (Shepard \& Jordan, 1984) as supporting the idea that the perception and memory of tones is mediated by a tonal schema with the following template-like properties: (1) It behaves as a semirigid structure with respect to the log-frequency continuum, although it may be subject to some small degree of stretching or compression to accommodate tonal sequences that are stretched or compressed in pitch. (2) It can, however, readily shift, as a whole, up and down that continuum, in order to achieve the best possible fit with tones in a particular musical key and can, accordingly, make slight (and perhaps unnoticed) shifts of this kind in order to maintain registration with tones that come in out of tune-for example, increasingly sharp, as in the case of our stretched ascending scale. (3) In shifting along the pitch continuum, the semirigid template preserves the characteristic intertone spacing ( 2 , $2,1,2,2,2,1)$ of the major diatonic scale. (4) This schema, when brought into register with incoming tones, also assigns musically significant tonal functions (tonic, dominant, mediant, leading tone, etc.) to the tones on the basis of their uniquely defined positions within the asymmetrically spaced schema. (5) When a proper registration is not attainable between the template and the incoming tones, because, for example, the pitch intervals between adjacent tones are equal (rather than unequal, as expected by the template), the schema may be imposed by default, giving rise to illusions or distortions in the perception and memory of those tones.

Perceptual schemas, which have long been recognized to be important in visual perception and cognition, appear to be of equal importance in the perception of musical tones. Moreover, just as the mental representation of structure-preserving transformations evidently underlies the comparison of visual objects, as in the phenomenon called mental rotation (Shepard \& Cooper, 1982; Shepard $\&$ Metzler, 1971), the mental representation of structurepreserving transformations (in this case, of the tonal template) may underlie the comparison of auditory objects.

\section{REFERENCES}

Attneave, F., \& Olson, R. K. (1971). Pitch as a medium: A new approach to psychological scaling. American Journal of Psychology, 84, 147-166.

BaLZANo, G. J. (1980). The group-theoretic description of twelvefold and microtonal pitch systems. Computer Music Journal, 4, 66-84.

BurNs, E. M., \& WARD, W. I. (1978). Categorical perceptionphenomenon or epiphenomenon: Evidence from experiments in the perception of melodic musical intervals. Journal of the Acoustical Society of America, 63, 456-468.

Castellano, M. A., Bharucha, J. J., \& Krumhansl, C. L. (1984). Tonal hierarchies in the music of North India. Journal of Experimental Psychology: General, 113, 394-412.

Chandere, J. P. (1969). STEPIT-Finds local minima of a smooth function of several parameters. Behaviorial Science, 14, 81-82.

Dowling, W. J. (1978). Scale and contour: Two components of a theory of memory for melodies. Psychological Review, 85, 341-354.

Dowling, W. J., \& Fujitani, D. S. (1971). Contour, interval, and pitch recognition in memory for melodies. Journal of the Acoustical Society of America, 49, 524-531.

JORDAN, D. S. (1987). Influence of the diatonic tonal hierarchy at microtonal intervals. Perception \& Psychophysics, 41, 482-488.

Kessler, E. J., Hansen, C., \& Shepard, R. N. (1984). Tonal schemata in the perception of music in Bali and in the West. Music Perception, 2, 131-165.

Krumhansl, C. L. (1985). Perceiving tonal structure in music. American Scientist, 73, 371-378.

Krumhanst, C. L. (1987). Perceptual structures for musical pitch. Monograph in preparation, Cornell University, Ithaca, NY.

KrumhansL, C. L., \& Kessler, E. J. (1982). Tracing the dynamic changes in perceived tonal organization in a spatial representation of musical keys. Psychological Review, 89, 334-368.

Krumhansi, C. L., \& ShePaRd, R. N. (1979). Quantification of the hierarchy of tonal functions within a diatonic context. Journal of Experimental Psychology: Human Perception \& Performance, 5, 579-594.

Sattath, S., \& Tversky, A. (1977). Additive similarity trees. Psychometrika, 42, 319-345.

SHEPARD, R. N. (1981). Psychophysical complementarity. In M. Kubovy \& J. R. Pomerantz (Eds.), Perceptual organization. Hillsdale, NJ: Erlbaum.

SHEPARD, R. N. (1982). Geometrical approximations to the structure of musical pitch. Psychological Review, 89, 305-333. 
ShePard, R. N., \& Cooper, L. A. (1982). Mental images and their transformations. Cambridge, MA: MIT Press/Bradford Books.

SHEPARD, R. N., \& JoRdaN, D. S. (1984). Auditory illusions demonstrating that tones are assimilated to an internalized musical scale. Science, 226, 1333-1334.

Shepard, R. N., \& METzler, J. (1971). Mental rotation of threedimensional objects. Science, 171, 701-703.

Siegel, J. A., \& Siegel, W. (1977). Absolute identification of notes and intervals by musicians. Perception \& Psychophysics, 21, 143-152.

ZATORRE, R. S., \& HALPERN, A. R. (1979). Identification, discrimination, and selective adaptation of simultaneous musical intervals. Perception \& Psychophysics, 26, 384-395.

APPENDIX A

Fourier Analysis of Experiment 1 Profiles

\begin{tabular}{crrrr}
\hline Scale & Harmonic & \multicolumn{1}{c}{$A_{k}$} & \multicolumn{1}{c}{$\Phi_{k}$} & \% Variance \\
Ascending & \multicolumn{3}{c}{ Group 1 Listeners } & \\
& 0 & 0 & 0 & 0 \\
& 1 & 0.24144 & -0.01594 & 0.07211 \\
& 2 & 0.27051 & -0.73093 & 0.09052 \\
& 3 & 0.25577 & -1.00960 & 0.08092 \\
& 4 & 0.21501 & -1.19373 & 0.05718 \\
& 5 & 0.44343 & -1.03839 & 0.24322 \\
& 6 & 0.03650 & 2.81623 & 0.00165 \\
& 7 & 0.26944 & 1.30111 & 0.08980 \\
& 8 & 0.18796 & -0.80091 & 0.04370 \\
& 9 & 0.26255 & -1.32829 & 0.08527 \\
& 10 & 0.15887 & -0.78907 & 0.03122 \\
& 11 & 0.03261 & -1.09131 & 0.00132 \\
& 12 & 0.40521 & 0 & 0.20310 \\
& 0 & 0 & 0 & 0 \\
& 1 & 0.25124 & 1.24287 & 0.07144 \\
& 2 & 0.37005 & -0.93259 & 0.15498 \\
& 3 & 0.18264 & 0.37650 & 0.03775 \\
& 4 & 0.30460 & 1.19103 & 0.10501 \\
& 5 & 0.38942 & -0.81150 & 0.17163 \\
& 6 & 0.11039 & -0.12108 & 0.01379 \\
& 7 & 0.35145 & 0.63240 & 0.13980 \\
8 & 0.08658 & -1.31288 & 0.00848 \\
& 9 & 0.17865 & 0.56390 & 0.03612 \\
& 10 & 0.22423 & -0.57062 & 0.05691 \\
& 12 & 0.06402 & 0.69480 & 0.00464 \\
& 0.41979 & 0 & 0.19945
\end{tabular}

Group 2 Listeners

Ascending

$\begin{array}{lrrrr} & 1 & 0.43520 & -0.65246 & 0.08501 \\ & 2 & 0.60678 & -0.56676 & 0.16526 \\ & 3 & 0.47307 & -0.43149 & 0.10045 \\ & 4 & 0.21886 & -0.68131 & 0.02150 \\ 5 & 0.76858 & -0.70832 & 0.26515 \\ & 6 & 0.11782 & 2.64789 & 0.00623 \\ & 7 & 0.59859 & 0.77908 & 0.16083 \\ & 8 & 0.23985 & 0.07530 & 0.02582 \\ & 9 & 0.09676 & 0.95203 & 0.00420 \\ & 10 & 0.18168 & 0.25947 & 0.01482 \\ & 11 & 0.01113 & 2.47915 & 0.00006 \\ & 12 & 0.57937 & 0 & 0.15067 \\ & 0 & 0 & 0 & 0 \\ & 1 & 0.29061 & 1.43246 & 0.02930 \\ & 2 & 0.32495 & -0.36810 & 0.03664 \\ & 3 & 0.75184 & -0.35158 & 0.19611 \\ & 4 & 0.24282 & 3.01344 & 0.02046\end{array}$

APPENDIX A (Continued)

\begin{tabular}{rrrrr}
\hline Scale & Harmonic & \multicolumn{1}{c}{$A_{\boldsymbol{k}}$} & \multicolumn{1}{c}{$\Phi_{\boldsymbol{k}}$} & $\%$ Variance \\
\cline { 2 - 5 } & 5 & 0.89523 & -0.82081 & 0.27805 \\
6 & 0.43718 & -1.52217 & 0.06631 \\
7 & 0.74094 & 0.45349 & 0.19047 \\
8 & 0.15379 & 1.37446 & 0.00821 \\
9 & 0.32254 & -0.07423 & 0.03609 \\
10 & 0.13451 & -0.05381 & 0.00628 \\
11 & 0.19924 & -0.69000 & 0.01377 \\
12 & 0.58396 & 0 & 0.11831 \\
\hline
\end{tabular}

Fourier equation:

$$
f(\theta)=\sum_{k=0}^{n \text { data } / 2} A_{k} \cos \left(k \theta-\Phi_{k}\right)
$$

Legend: $f$ is the estimated Fourier curve over the variable $\theta$, $n$ data $=24$ is the number of data points, $A_{k}$ is the amplitude of the $k$ th harmonic in the curve, $\Phi_{k}$ is the phase (in radians) of the $k$ th harmonic in the curve, and \% Variance is the percent of variance explained by the kth harmonic.

\section{APPENDIX B}

Fourier Analysis of Experiment 2 Profiles

\begin{tabular}{crrrr}
\hline Scale & Harmonic & \multicolumn{1}{c}{$A_{k}$} & \multicolumn{1}{c}{$\Phi_{k}$} & $\%$ Variance \\
Ascending & \multicolumn{3}{c}{ Group 1 Listeners } & \\
& 0 & 0 & 0 & 0 \\
& 1 & 0.36542 & -0.17025 & 0.03203 \\
& 2 & 0.49833 & -0.11064 & 0.05957 \\
& 3 & 0.87417 & -0.32894 & 0.18330 \\
& 4 & 0.42833 & 0.52992 & 0.04401 \\
& 5 & 0.90439 & -1.18538 & 0.19619 \\
& 6 & 0.23792 & 0.82132 & 0.01358 \\
& 7 & 0.96381 & 1.00982 & 0.22282 \\
& 8 & 0.15212 & -0.90678 & 0.00555 \\
& 9 & 0.24093 & -0.18390 & 0.01392 \\
& 10 & 0.21170 & -0.18598 & 0.01075 \\
& 11 & 0.20022 & -0.79343 & 0.00962 \\
& 12 & 0.93271 & 0 & 0.20867 \\
& 0 & 0 & 0 & 0 \\
& 1 & 0.15563 & 1.37108 & 0.00517 \\
& 2 & 0.38759 & -0.70859 & 0.03206 \\
& 3 & 0.52899 & -0.28142 & 0.05973 \\
& 4 & 0.56599 & -1.45496 & 0.06837 \\
& 5 & 1.06387 & -0.95679 & 0.24157 \\
& 6 & 0.33314 & 1.23164 & 0.02369 \\
& 7 & 1.15195 & 0.72635 & 0.28323 \\
& 8 & 0.19577 & -0.70118 & 0.00818 \\
& 10 & 0.47864 & 0.31456 & 0.04890 \\
& 11 & 0.04808 & -0.44200 & 0.00049 \\
& 12 & 0.99083 & 0 & 0.20954
\end{tabular}

Ascending

Group 2 Listeners

$\begin{array}{llll}0 & 0 & 0 & 0 \\ 1 & 0.59480 & -0.97454 & 0.22685 \\ 2 & 0.46473 & -1.10971 & 0.13849 \\ 3 & 0.38305 & -0.43013 & 0.09408 \\ 4 & 0.28043 & -1.08710 & 0.05043 \\ 5 & 0.68291 & -0.67256 & 0.29904 \\ 6 & 0.06333 & -0.65008 & 0.00257\end{array}$


APPENDIX B (Continued)

\begin{tabular}{ccccc}
\hline Scale & Harmonic & \multicolumn{1}{c}{$A_{k}$} & \multicolumn{1}{c}{$\Phi_{k}$} & $\%$ Variance \\
\hline & 7 & 0.05779 & -0.31567 & 0.00214 \\
& 8 & 0.10418 & 1.38984 & 0.00696 \\
& 9 & 0.12722 & -0.69982 & 0.01038 \\
Descending & 10 & 0.21116 & -0.99429 & 0.02859 \\
& 11 & 0.25656 & 0.32172 & 0.04221 \\
& 12 & 0.39146 & 0 & 0.09826 \\
& 1 & 0 & 0 & 0 \\
& 2 & 0.60823 & 0.60540 & 0.27993 \\
& 3 & 0.47036 & -0.90803 & 0.16741
\end{tabular}

APPENDIX B (Continued)

\begin{tabular}{rrrrr}
\hline Scale & Harmonic & \multicolumn{1}{c}{$A_{k}$} & \multicolumn{1}{c}{$\Phi_{k}$} & $\%$ Variance \\
\hline 4 & 0.38601 & 0.08611 & 0.11275 \\
5 & 0.56439 & -0.52002 & 0.24103 \\
6 & 0.08342 & -1.52583 & 0.00527 \\
7 & 0.23276 & 1.43565 & 0.04099 \\
8 & 0.10294 & 0.31360 & 0.00802 \\
9 & 0.17500 & 1.02112 & 0.02317 \\
10 & 0.10789 & 1.02380 & 0.00881 \\
11 & 0.04440 & -0.46883 & 0.00149 \\
12 & 0.30354 & 0 & 0.06972 \\
\hline
\end{tabular}

Note-For Fourier equation and legend, see Appendix A. 